\title{
A Survey of Morphing Techniques
}

\author{
Bhushan Zope ${ }^{1}$, Soniya B. Zope ${ }^{2}$ \\ ${ }^{1}$ Pune Institute of Computer technology, Pune University, Pune, Maharashtra, India \\ ${ }^{2}$ AISSMS College of Engineering, Pune University, Pune, India
}

\begin{abstract}
Image morphing provides the tool to generate the flexible and powerful visual effect. Morphing depicts the transformation of one image into another image. The process of image morphing starts with the feature specification phase and then proceeds to warp generation phase, followed by the transition control phase. This paper surveys the various techniques available for all three stages of image morphing.
\end{abstract}

Keywords - Image morphing, Feature specification, Warp generation, Transition control.

\section{INTRODUCTION}

Morphing is defined as a process which changes the one image into other through seamless transition. Thus it is the fluid visual transformation of one image into another image using the sequence of intermediate images. It is often used to create the special effects in movies such as Terminator, Willow and Indiana Jones and the last crusade, in music videos such as Michael Jackson's Black or White [31]. Morphing is also used in the gaming industry to add engaging animation to video games and computer games. However, morphing techniques are not limited only to entertainment purposes. Morphing is a powerful tool that can enhance many multimedia projects such as presentations, education, electronic book illustrations, and computer-based training.

In morphing there are a number of intermediate images that are generated while transforming source image to destination image. Initial intermediate image is very similar to source image while last intermediate image is similar to destination image. Middle intermediate image is the average of source image distorted halfway towards destination and the destination image distorted towards the source. Any intermediate image contains the feature of both images. The degree of similarity of that image depends on its position in the intermediate image sequence.

Morphing process between two images is initiated with the specification of the correspondence points. This phase is usually called as 'feature specification'. The feature specification process defines the corresponding feature primitives for both images. This process is the most tedious aspect of morphing and performed manually in most cases. Each primitive denotes the image landmark or feature. Corresponding feature sets are then used to compute the mapping function, which is called as 'warp function'. This warp function calculates and transforms each pixel in one image to new position in other image. Thus it specifies the relation between pixels in both images. This brings the last phase of morphing: transition control. Transition control blends in the color between both images and also decides the rate of warping [30]. Transition control is responsible of smooth and fluid transformation of images. Thus morphing is a three phase method viz. Feature specification, warp generation, and transition control.

\section{FEATURE SPECIFICATION AND WARP GENERATION}

Feature Specification and Warp Generation problem has been studied in many contexts. Feature specification can be achieved by various techniques like, Mesh based warping [31], feature based constraints [4], energy minimization [17] and radial basis function [1]. Feature can also be specified using line segments, curves, points and snakes [32]. Features can be automatically detected and extracted using FDNN and filters [21]. Once the features are specified the mapping between them can be obtained using bilinear mapping, spline-based mapping or free form deformation [32], triangle coordinate system [26], triangulation method [12].

In following sub-section we will review some of the available techniques for feature specification and warp generation.

\subsection{LINEAR IMAGE MORPHING}

This is the simpler method of morphing. In Linear Morphing, linear images are segmented into lines and then transforming them according to the target image. [21].

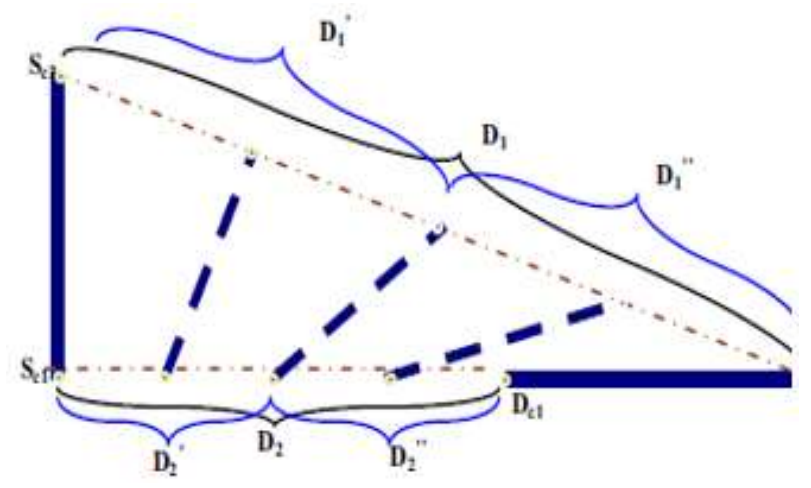

Fig. 1: Linear Morphing 
Transformation of source image into the target image is clearly visible in Fig. 1. Here the end points of the lines are detected first and then generating the mid points of the distance of the corresponding end points of the source and target line images, and then drawing the intermediary lines in between the distances of the end points. This is most effective for the linear images but quite impossible to morph an image like facial or other figures.

\subsection{MESH WARPING}

Mesh warping was pioneered at Industrial Light \& Magic (ILM) by Douglas Smythe for use in the movie Willow in 1988. This technique is well described in wolberg's book [31]. Source and the target images have meshes associated with them. Mesh of the source image specifies the coordinates of control points, or landmarks. The second mesh of the target image specifies their corresponding positions in the target image. The meshes are respectively overlaid on source and target images. Landmarks such as the eyes, nose, and lips lie below corresponding grid lines in both meshes. Together, the meshes are used to define the spatial transformation that maps all points in source image onto target image. The use of meshes for feature specification facilitates a straightforward solution for warp generation: bicubicspline interpolation but while meshes appear to be a convenient manner of specifying pairs of feature points, they are, however, sometimes cumbersome to use.

Thus consider $I_{s}$ and $I_{d}$ as the source and destination images respectively. $M_{S}$ is the mesh corresponding to $I_{S}$ whereas $M_{d}$ is the mesh associated with $I_{d}$. Thus $M_{s}$ and $\mathrm{M}_{\mathrm{d}}$ are going to help the transformation from $\mathrm{I}_{\mathrm{s}}$ to $\mathrm{I}_{\mathrm{d}}$. All intermediate images are produced using 4 step process, explained in [30]:

For each frame $f$ do

Linearly interpolate mesh $\mathrm{M}$, between $\mathrm{M}_{\mathrm{s}}$ and

$\mathrm{M}_{\mathrm{d}}$

Warp $\mathrm{I}_{\mathrm{s}}$ to $\mathrm{I}_{1}$, using meshes $\mathrm{M}_{\mathrm{s}}$ and $\mathrm{M}$

Warp $I_{d}$ to $I_{2}$ using meshes $M_{d}$ and $M$

Linearly interpolate $\mathrm{I}_{\mathrm{f}}$, between $\mathrm{I}_{1}$ and $\mathrm{I}_{2}$

End

In mesh warping [31], features are specified by a nonuniform control mesh, and a warp is computed by a spline interpolation.

There are various approaches of mesh warping depending upon how the mesh is implemented.

\subsubsection{USING BEZIER CLIPPING}

Nishita et al.[20] proposed the new mesh warping technique using a non uniform control mesh to specify features and computed a warp using a two-dimensional free form deformation and the Bezier clipping. This method is extension to mesh warping. Easy manipulation of mesh setting by the automatic contour extraction of images can be realized using this technique. In this technique, the mesh specified is treated as the Bezier net. This net is the two dimensional elastic network model which minimizes the energy functional. Its energy functional is nothing but internal strain energy and image energy . this energy attracts the net to features. The net deforms to warp the images as the energy functional is minimized. This method specifies the speed of transformation by a Bezier function defined on the mesh.

\subsubsection{TRIANGLE MESH WARPING}

Another approach to mesh warping is the triangular mesh warping proposed by Ruppert [23]. In triangle mesh warping, the image is divided into set of suitable triangles with the specified data points being the corner of the triangle. In this method triangulation method is carried out so that the points inside the triangle are closer to its own vertices than to vertices of other triangles. The intersection of any two triangles is a common edge, a common vertex, or the empty set.

Mesh warping shows a good distortion behavior but has a critical drawback in specifying features. It always require a control mesh on an image while its features may have an arbitrary structure .Mesh warping controls the transition behavior with a mesh used for specifying features. Mesh warping assigns a transition curve for each point of the mesh, and the curves determine the transition rate in interpolating the position of each point. It is also time consuming to define the feature correspondence via a user interface. Also the number of steps required in mesh morphing is relatively large. This large number of steps make algorithm run slowly. The warp generation function is very complicated and this makes the programming hard. In mesh warping method, points may be left unmodified by mistake or there will not be any corresponding point for a particular point. Such points are also used in the warping function. Due to this the animator sometimes feels that he has no control in some places.

\subsection{FIELD MORPHING}

The field morphing algorithm is developed by Beier and Neely [4] at Pacific Data Images. In this technique, features are specified with the line segments. These line segments impose the influence on the pixels nearby. The pixels are then warped according to their proximity with the line segments. A pair of corresponding lines in the source and target images defines a coordinate mapping between the two images. In addition to the straightforward correspondence provided for all points along the lines, the mapping of points in the vicinity of the line can be determined by their distance from the line. In the case of multiple line pairs, the warping of a given point is calculated by a weighted sum of mappings of all 
line pairs. This method is easy to use to specify corresponding features.

The transformation is given by the following formulas [4] The coordinate mapping $\mathrm{u}$ and $\mathrm{v}$ are:

$$
\begin{aligned}
& \mathrm{u}=(\mathrm{X} 1-\mathrm{P} 1) \cdot(\mathrm{Q} 1-\mathrm{P} 1) /\|\mathrm{Q} 1-\mathrm{P} 1\| 2 \\
& \mathrm{v}=(\mathrm{X} 1-\mathrm{P} 1) \cdot \operatorname{Perp}(\mathrm{Q} 1-\mathrm{P} 1) /\|\mathrm{Q} 1-\mathrm{P} 1\|
\end{aligned}
$$

The value $\mathrm{u}$ is the position along the oriented line P1Q1 and $\mathrm{v}$ is the distance from this line for each pixel $\mathrm{X} 1$ of the intermediate image. Perp is the vector perpendicular to the given vector.

The calculation of $\mathrm{X} 2$ in the source image and $\mathrm{X} 3$ in the destination image using $\mathrm{u}$ and $\mathrm{v}$ are given by using the given formula.

X2 P2 u.(Q2 P2) (v.= + - + Perp(Q2 - P2/ || Q2-P2 |)

$\mathrm{X} 3=\mathrm{P} 3+\mathrm{u} \cdot(\mathrm{Q} 3-\mathrm{P} 3)+(\mathrm{v} \cdot \mathrm{Perp}(\mathrm{Q} 3-\mathrm{P} 3 /\|\mathrm{Q} 3-\mathrm{P} 3\|)$

To specify many features in image, we have more complex transformation, which include a weighted combination of the transformation performed by each line pair. Therefore, the weight of each pair is computed as follows:[4]

Weight $=[($ length $) \mathrm{p} /(\mathrm{a}+\mathrm{dist})] \mathrm{b}$

length $=$ The length of a vector

dist $=$ The distance from pixel to the vector

$\mathrm{a}, \mathrm{b}, \mathrm{p}=$ Constants can be used to change the relative effect of the vectors

While the program is straightforward and fun to use, it still requires a lot of work from the animator. It is much more expressive. The only positions that are used in the algorithm are ones the animator explicitly created. This approach has the benefit of being more expressive than mesh warping. For example, rather than requiring the correspondence points to all lie on a mesh, line pairs can be drawn along the mouth, nose, eyes, and cheeks of the source and target images. Therefore only key feature points need to be given. Although this approach simplifies the specification of feature correspondence, it complicates warp generation. The two biggest disadvantages of feature-based technique are speed and control. This technique is slower than mesh warping. This is due to the fact that all line pairs must be considered before the mapping of each source point is known, while mesh warping uses bicubic interpolation to determine the mapping of all points not lying on the mesh. A more serious difficulty, though, is that unexpected displacements may be generated after the influence of all line pairs are considered at a single point. This result in the appearance of a part of the image in unrelated regions in the in-between images (often referred to as "ghosts"). Additional line pairs must sometimes be supplied to counter the ill effects of a previous set. The mesh warping and field morphing algorithms have both been used to produce startling visual effects.

\subsubsection{MODIFIED FEATURE-BASED MORPHING}

[11] Try to optimize the feature-based image morphing technique. Hussain Karam et al. propose two algorithms for the optimization. First algorithm, End-points algorithm, slightly modifies Beier's to transform featurelines. Here only end points of feature line are morphed and remaining points are transformed according to these end points. This modification speed up the Beier's algorithm. Second algorithm proposed in [11] is Local Decomposition Algorithm, which uses the decomposition methodology. Input images are subdivided into regular grid regions. Grid size depends upon the linearity of image. In the highly linear region, simple linear approximation can generate the good morph, which reduces the computation time.

\subsubsection{USING FEATURE CURVES}

H. Birkholz and D. jackel's approach [5] can be considered as an extension to method of Neely and Beier. Instead of using vectors curves are used to mark the features in source and destination objects. So due to use of curves, the number of curves required are reduced as compare to number of vectors. So the chance of ghost image appearing becomes less. The combination of curves and vectors can generate the precise warps.

\subsubsection{USING BEZIER CURVES AND DEPENDENCY GRAPH}

H. Johan et al. proposes the another approach to feature based morphing in [10]. They represented the features with Bezier curves and the connection between them is represented with the dependency graph. Feature interpolation is performed by interpolating the dependency graph using the edge-angle blending technique. The end points of the feature curves are represented as the node of the graph while a edge between two nodes represents the directed line segment connecting the its two nodes. Thus there is a dependency graph for each input and the intermediate features are generated by applying edge-angle technique between these two graphs.

\subsection{SCATTERED DATA INTERPOLATION}

In all the algorithms explained till now, feature primitives are specified using points, lines and curves. But it can be specified by a set of points, as lines and curves can be point sampled. Thus the warp can be generated by constructing the surface that interpolates these scattered points. Consider, for example set of feature points labeled $\left(\mathrm{u}_{\mathrm{k}}, \mathrm{v}_{\mathrm{k}}\right)$ in source image and $\left(\mathrm{x}_{\mathrm{k}}, \mathrm{y}_{\mathrm{k}}\right)$ in destination image, where $1<=\mathrm{k}<=\mathrm{M}$. Deriving warp functions that map points from the target image to the source image is equivalent to determining two smooth surfaces: one that passes through points $\left(\mathrm{x}_{\mathrm{k}}, \mathrm{y}_{\mathrm{k}}, \mathrm{u}_{\mathrm{k}}\right)$ and the other that passes 
through $\left(\mathrm{x}_{\mathrm{k}}, \mathrm{y}_{\mathrm{k}}, \mathrm{v}_{\mathrm{k}}\right)$ for $1<=\mathrm{k}<=\mathrm{M}$. In this way the warp generation problem can be addressed by scattered data interpolation. Scattered data interpolation approach can be found in the work of Ruprecht and M"uller [22] that provides smooth warping combined with an easily controllable behavior.

The scattered data interpolation is achieved through various techniques.

\subsubsection{USING RADIAL BASIS FUNCTION}

Another scattered data interpolation method using radial basis function was introduced by Arad and Reisfeld [1]. Arad and Risefeld use so-called anchor points to control the warping process and construct the warping function as a linear combination of radial basis function. Radial basis functions guarantee a smooth surface deformation from a sparse set of control points. However, if a control point is moved too far from its original position, say outside the influence region, large discontinuities occur around the anchor points. Because anchors points are stationary at the boundary of the influence region, no influence of the control point will propagate through the anchor points [32].

\subsubsection{USING DEFORMABLE SURFACES}

Two similar methods were independently proposed using the thin plate surface model $[16,19]$. Multigrid relaxation method is used to compute deformable surfaces for warp generation or transition function. This multigrid relaxation method makes this technique fast and the computation time remains nearly constant even if the number of control points increases.

Techniques depending on scattered data interpolation, gives freedom to animator as feature primitives are specified by an arbitrary point set. These techniques also generate smooth warps that exactly reflect the feature correspondence. Furthermore, they offer the most general form of feature specification since any primitive (e.g., spline curves) may be sampled into a set of points.

\subsection{ENERGY MINIMIZATION}

One to one property of the generated warp prevents the warped image from folding back to itself. But all the methods described above do not satisfy such property. An energy minimization method has been proposed for generating one to one warp function in [17].

This technique gets warping function simulating square rubber membrane. The square rubber membrane can change its shape because of pushpin. If we move pushpin of rubber membrane, the rubber membrane is changed. The location and corresponding relation of feature dot are like pushpin. This technique observes the feature of rubber membrane that is the location restriction of pushpin, presents the feature that meets one to one correspondence and is successive into energy clause, and calculates warping function minimizing the sum of it.
Because energy minimization technique calculates physically meaningful energy clause, transform is natural and it generates warping function that is one to one correspondence. But when we calculate warping function, we need much time. Also it has high computational cost [30].

\subsection{MULTILEVEL FREE-FORM DEFORMATION}

A new warp generation method was presented in [15] that is much simpler and faster than the related energy minimization method in [17]. Large performance gains are achieved by applying multilevel free-form deformation (MFFD) across a hierarchy of control lattices to generate one-to-one and $\mathrm{C}^{2}$-continuous warp function. MFFD is morphing technique of expanded FFD concept, and appoints the feature with dots. In order to move the point on source image to its corresponding point on destination image, the behavior of movement of lattice points around the point is calculated and then plate is moved accordingly. The calculation speed of this method is faster than energy minimization technique's, and this method calculates warping function that satisfies one to one correspondence.

One important benefit of MFFD-based morphing is that feature specification is more expressive and less cumbersome. Rather than editing a mesh, for instance, only a small set of features must be specified. To further assist the user, snakes can be introduced to reduce the burden of feature specification. Snakes streamline feature specification because primitives must only be positioned near the features. Image forces push snakes toward salient edges, thereby refining their final positions and making it possible to capture the exact position of a feature easily and precisely.

The MFFD algorithm can be combined with energy minimization method to produce new hybrid approach. This hybrid approach produces the nice warps similar to energy minimization method in computation time comparable to MFFD method.

\subsection{AUTOMATICALLY SPECIFIED FEATURES}

All above methods needs the feature primitives to be specified manually. But S. Karungaru et al. [2] presented a method using which the face images can be warped and morphed automatically. Feature specification is done automatically using face detection neural network (FDNN), edge detection and smoothing filters. FDNN extracts the feature from an image with the 97\% [12]. These control points are extracted for both the source and destination images. Filters are used to confirm these positions. Once these points are found, the warping is done using triangle based mesh morphing, which is explained earlier. The color transition is the method which determines the rate of color blending across the 
sequence and it is performed using the one-dimension Gaussian function.

The images can be automatically morphed with this method which reduces the input needed to be entered by user. This method uses only 5 points to generate good result. This method saves lot of computational time as the control points required to morph the images is very less. Only face images can be morphed using this method is the disadvantage of it.

Above all methods require the feature specification, either manual or automatic, but there are some other methods which don't require any specification.

\subsection{BASED ON PIXEL TRANSFORMATION}

M. T. Rahman et al.[21] presents the morphing method based on the pixel transformation. Pixel-based morphing is achieved by replacement of pixel values followed by a simple neighboring operation. The replacement of pixel value of source image is done by convolving the neighbor with the help of mask. The pixels of source image and destination images are divided into 3 categories.

C1: pixels those are white in target image not in source image.

C2: pixels those are white in source image not in target image.

C3: the pixels those are of any other gray level in target image.

After categorizing the pixel in images, the white pixels are transformed from one side and gray from other side. Pixels are rearranged according to target image.

This method is very simple and efficient for morphing and the transformation is performed according to the neighborhood of pixels so no abrupt changes are found.

\subsection{BASED ON OPTIMAL MASS PRESERVING MAPPING}

L. Zhu et la. [33] proposes an algorithm based on the theory of optimal mass transport. Warping is done with the help of pixel intensities and A successful intensitybased algorithm can achieve automatic morphing without user inputs or prior assumptions on special shapes or features of the objects in the image. In this technique, intensity is considered as mass density and applying optimal mass transportation algorithm on two related images, deformation grid is generated.

\subsection{REGENERATIVE MORPHING}

Eli Shechtman et al. [25] present a new image morphing approach in which the output sequence is regenerated from small pieces of the two source (input) images. In this method morphing is viewed as a optimization problem with an objective function that captures source similarity and temporal coherence. The approach does not require manual correspondence, and generates compelling results even when the images are of very different objects. The temporal coherence directly minimizes ghosting and blurring effect of morphing and automatically generates the interesting morph patterns from two unrelated images.

\section{COLOR TRANSITION}

A morph contains a sequence of intermediate images from the source image to the target image. Color transition is the method that determines the rate of color blending across the sequence. The choice of this rate determines the quality of the morphs. Interesting morphs can be created depending on whether the color-blending rate changes locally or globally.

Some of the color blending algorithms is explained in following subsection.

\subsection{CROSS DISSOLVE}

In the times when morphing is not yet developed, image transformation is carried out with the help of cross dissolve technique. Cross dissolve is the linear interpolation to fade from one image to another [30]. According to cross dissolve approach, in order to make an intermediate frame we align the geometry, and superpose the two images. The color value particular pixel of intermediate frame $\left(\mathrm{I}_{\mathrm{f}}\right)$ is dependent on the color value respective pixel in source image $\left(\mathrm{I}_{\mathrm{s}}\right)$ and destination image $\left(I_{d}\right)$. This can be calculated with the formula:

$I_{f}(i, j)=I_{s}(i, j) * v+I_{d}(I, j) *(1-v)$ where $v \in[0,1]$.

The result of cross dissolve method is poor, owing to the double-exposure effect apparent in misaligned regions. This problem is particularly apparent in the middle frame, where both input images contribute equally to the output [30]. Image at the half way of morphing process may be seemed to be a ghost effect or a gloomy zigzag image. Towards the target image and near source image it is little clear but seems like another image is rising up from the background. Warping maintains the geometric alignment of these regions throughout the cross dissolve process, hence morphing achieves the fluid transformation between source and destination image.

\subsection{ONE-DIMENSIONAL GAUSSIAN FUNCTION}

The rate of color blending is decided by calculating the weight. The one method to calculate weight is onedimensional Gaussian function. The color difference between source and target image is calculated and depending upon the difference, the color of new pixel is calculated with following formula:

$$
\boldsymbol{\beta} \mathbf{j}=\boldsymbol{\beta} \mathbf{i}-\boldsymbol{\alpha i} * \Delta \boldsymbol{\alpha} \mathbf{i j}
$$

Where $\beta \mathrm{j}$ is the color for the new pixel in an intermediate image, $\alpha \mathrm{i}$ is the weight and $\Delta \alpha \mathrm{ij}$ is the color difference between the target and source pixels. This practice is recurring for every pixel in the image and for every intermediate image in the morph sequence. Finally the source image slowly changes to target image. Despite the fact that this method produces good morphs, it takes large computation time. 
3.3 PIXEL BASED COLOR TRANSITION

H. B. Kekre et al. [13] proposes the extension to cross dissolve. In this method weights required to control the rate of blending are computed using difference in the color of two corresponding pixels from source and target images and the number of intermediate frames required. Thus step size computation requires proper adjustments of weight according to number of frames required. Computation of weights and deciding step size is simple and can be done in faster manner. Also generating intermediate image requires simple addition operation. The quality of morphs is good and hence when represented one after other as an animation seamless transition of two images than the cross dissolve is seen [13].

\section{CONCLUSION}

This paper surveyed the various morphing techniques available. All morphing algorithm has the three components: feature specification, warp generation, and transition control. The ease, with which an animator can produce the morph sequence and quality of that sequence, depends on how these components are addressed in the algorithm.

The earliest morphing algorithm is linear morphing. It is the simplest morphing method but this method is quite impossible to morph facial images. Mesh warping is another method for morphing, in which features are specified by a non-uniform control mesh, and a warp is computed by a spline interpolation. This approach is straightforward but large number of steps in mesh warping make algorithm runs slowly. The warp generation function is very complicated and this makes the programming hard. Field morphing algorithm simplifies the feature specification with the use of feature line pair. Warp generation is complex in field morphing. The biggest disadvantage in this algorithm is appearance of ghost image in some cases. Warp generation is simplified using scattered data interpolation method. Standard methods like radial basis functions and thin plate splines have been demonstrated. Energy minimization technique provides the one to one mapping warp function which prevents the morph image to fold back to original image. This technique generates the smooth warps but its performance is affected by its high computational cost. A multilevel free-form deformation technique further accelerates the warp generation. The use of snake assists the user in placing feature primitives. It reduces the burden in feature specification.

\section{REFERENCES}

[1] Arad, N., Dyn, N., Reisfeld, D. and Yeshurun, Y,. 1994. Image warping by radial basis functions:
Applications to facial expressions, CVGIP: Graphical Models and Image Processing, vol. 56(2), pp. 161-172.

[2] Alexa, M. \& Darmstadt, T.U.,2001. Local Control for Mesh Morphing, International Conference on Shape Modeling and Applications, SMI 2001, pp. $209-215$.

[3] Bagade, A. M. and Talbar, S. N., 2010. Image Morphing Concept for Secure Transmission of Image Data Contents over Internet, Journal of Computer Science, vol. 6(9), pp. 987-992.

[4] Beier, T. and Neely, S., 1992. Feature-based image metamorphosis, Proc. Computer Graphics, vol. 26(2), pp. 35-42.

[5] Birkholz, H. And Jack, D., 2003. Image Warping with Feature Curves, Proc. spring conference on Computer graphics, pp. 199-202.

[6] Chalidabhongse, J. \& Kuo, C.-C. J., 1993. A multiresolution approach for image morphing, Proc. of 27th Asilomar Conference on Signals, Systems and Computers, (Ilm), pp.16-20.

[7] Glassner, A., 2003. DMorph, IEEE Computer Graphics and Applications, Vol. 23(5), pp.78-83.

[8] Shi-Min Hu, Chen-Feng Li, Hui Zhang, 2004. Actual Morphing: A Physics-Based Approach to Blending. Symposium A Quarterly Journal In Modern Foreign Literatures, pp. 2-7.

[9] Iwanowski, M.,2002. Image morphing based on morphological interpolation combined with linear filtering, J. WSCG.

[10] Johant, H., Koisot, Y., Nishitat, T., 2000. Morphing Using Curves and Shape Interpolation Techniques, The Eighth Pacific Conference on Computer Graphics and Applications, Proceedings, pp. 348359.

[11] Karam, H., Hassanien, A. and Nakajima, M., 2001. Feature-based image metamorphosis optimization algorithm, Proceedings Seventh International Conference on Virtual Systems and Multimedia, pp. 555-564.

[12] Karungaru, S., Fukumi, M. and Akamatsu, N., 2004. Morphing face images using automatically specified features, 46th Midwest Symposium on Circuits and Systems, vol. 2, pp. 741-744.

[13] Kekre, H. B., Sarode, T. K., \& Patil, S.M., 2011. A Novel Pixel Based Color Transition Method for 2D Image Morphing, Proc. ICWET, pp. 357-362.

[14] Kouzani, A. Z., Nahavandi, S., Kouzani, N., Kong, L. X., and She, F. H., 2000. A morphing technique for facial image representation, Conf. Proc. SMC, pp. 1378-1383.

[15]Lee, S.-Y., Chwa, K.-Y., Shin, S. Y. and Wolberg, G.,1995. Image metamorphosis using snakes and 
free-form deformations, Computer Graphics (Proc. SIGGRAPH '95), pp. 439-448.

[16]Lee, S.-Y., Chwa, K.-Y., Hahn, J., and Shin, S. Y., 1994. Image morphing using deformable surfaces, Proc. Computer Animation '94, pp 31-39.

[17] Lee, S.-Y., Chwa, K.-Y., Hahn, J., and Shin, S. Y., 1996. Image Morphing Using Deformation Techniques, The Journal of Visualization and Computer Animation, vol. 7(1), pp. 3-23.

[18]Lee, S.-Y., Chwa, K.-Y., Shin, S. Y., Wolberg, G., yong, K., 1996. Image Metamorphosis with Scattered Feature Constraints, IEEE Transactions on Visualization and Computer Graphics, Vol. 2(4), pp. $337-354$.

[19] Litwinowicz, P. and Williams, L., 1994. Animating images with drawings, Proc conference on Computer graphics and interactive techniques SIGGRAPH '94, pp. 409-412.

[20] Nishita, T., Fujii, T., and Nakamae, E., 1993. Metamorphosis using B'ezier clipping, Proc. First Pacific Conf. on Computer Graphics and Applications, pp. 162-173.

[21] Rahman, M. T., Al-Amin, M., Bakkre, J. B., Chowdhury, A. R., and Bhuiyan, M. A.,2007. A novel approach of image morphing based on pixel transformation, 10th International Conference on Computer and Information Technology, pp.1-5.

[22] Ruprecht, D. \& Muller, H., 1995. Image Warping with Scattered Data Interpolation, IEEE Computer Graphics And Applications, vol. 15(2), pp. 37-43.

[23] Ruppert, J., 1995. A Delaunay Refinement Algorithm for Quality 2-Dimensional Mesh Generation, presented at J. Algorithms, pp.548-585.

[24] Sederberg, T. W., and Parry, S. R., 1986. Free-form deformation of solid geometric models, Computer Graphics (Proc. SIGGRAPH '86), vol. 20(4), pp. 151-160.

[25] Shechtman, E., Rav-Acha, A., Irani, M. and Seitz, S.M., 2010. Regenerative morphing, Proc. CVPR, pp.615-622.

[26] Shoujue, W., 2011. A Novel Math Method of Image Morphing, 3rd International Conference ICCRD, Vol. 4, pp. $125-128$.

[27] Su, M.-C. and Liu, I.C., 1969. Facial Image Morphing by Self-organizing Feature Maps, International Joint Conference on Neural Networks, pp. 1969-1972.

[28]Tao, N. \& Long, D., 2004. A novel approach for image morphing, proc. IEEE Asia-Pacific Conference on Circuits and Systems, vol.1, pp. 97100.

[29] Whitaker, R.T.,2000. A level-set approach to image blending, IEEE transactions on image processing : a publication of the IEEE Signal Processing Society, 9(11), pp. 1849-61.

[30] Wolberg, G., 1996. Recent advances in image morphing, Proceedings of CG International '96, pp. 64-71.

[31] Wolberg, G., 1990. Digital ImageWarping.IEEE Computer Society Press, Los Alamitos, CA.

[32] J.-yongNoh, Fidaleo, D. \& Neumann, U., 2000. Animated deformations with radial basis functions, Proc. of the ACM symposium on Virtual reality software and technology, pp. 166-174.

[33]Zhu, L., Yang, Y., Haker, S., \& Tannenbaum, A., 2007. An image morphing technique based on optimal mass preserving mapping, IEEE transactions on image processing, vol. 16(6), pp. 1481-95. 